\title{
Mouse genomics programs and resources
}

\author{
The mouse: pushing the boundaries
}

\author{
Maja Bucan · Janan T. Eppig · Steve Brown
}

Published online: 12 September 2012

(C) Springer Science+Business Media, LLC 2012

This year we celebrate 10 years since the publication of the draft genome sequence of the C57BL/6J mouse inbred strain. Initial and ongoing comparative sequence analysis continues to highlight the mouse as a leading model for studying human biology and disease. The availability of the reference sequence transformed the efforts of individual laboratories to understand the function of a specific gene(s) by studying mouse mutants. Moreover, the mouse genetic community recognized the opportunity to build on and take advantage of the mouse genome sequence for the generation of large functional genomic resources that will enable the elucidation of entire pathways and biological systems. As a result, laboratories and programs around the world have developed a tremendously rich genetic toolbox. The focus of this special issue of Mammalian Genome is on these programs and resources that underpin an often spectacular acceleration in novel insights into biology and disease.

The complete genome sequence of the C57BL/6J inbred strain along with the initial gene annotation revolutionized our ability to link the genome sequence to phenotypic diversity by facilitating genetic screens. The sequence offered a roadmap for the generation of loss-of-function alleles by gene-based mutagenesis. Also, the painstaking work of identifying altered gene sequence in mutants from

\section{Bucan ( $\square)$}

Department of Genetics, Perlman School of Medicine,

University of Pennsylvania, Philadelphia, PA, USA

e-mail: bucan@mail.med.upenn.edu

\section{J. T. Eppig}

The Jackson Laboratory, Bar Harbor, ME, USA

S. Brown

MRC Mammalian Genetics Unit, MRC Harwell OX11 ORD, UK phenotype-based screens was reduced to sifting through a list of annotated genes in a critical interval. However, having a reference sequence for only one inbred strain is very limiting. For decades, the biomedical community has explored the rich biology and phenotypic diversity of many inbred strains and their combined genomes. A recent report on the whole genome sequence of 17 inbred strains explored sequence variation for both single nucleotide and structural variants, significantly illuminating genetic variation and associated phenotype variation, as well as throwing light on inbred strain origin (Keane et al. 2011). In this issue, Yalcin et al. describe these exciting findings and Simon et al. focus on the application of next-generation sequencing methods (exome and whole genome sequence) to mutation discovery.

Detailed knowledge of the spatial and temporal expression patterns in the developing mouse embryo and in adult tissues is critical for deciphering complex regulatory networks and helping to define the genetic architecture of systems. The results of numerous genome-wide expression studies using microarray technologies and in situ hybridization are assembled in several data collections. Sophisticated molecular, microscopic and computational approaches were applied to create freely available databases and digital atlases with rigorously annotated expression patterns (Armit et al., Geffers et al., Henry \& Hohmann). How to combine expression data from several sources and integrate them with other gene-centric and biological data resources is a major challenge as discussed by Ringwald et al.

Analysis of mutants represents a key step towards our understanding of how information encoded by the genome leads to specific phenotypes. Undoubtedly a very significant milestone in mouse genetics is the current availability and access of mouse mutants. The mouse genetics community has for many years invested heavily in animal 
facilities and repositories to handle and disseminate large collections of frozen embryos or sperm for the growing mouse mutant resource (see Donahue et al.). Moreover, there has been a parallel commitment to developing and improving the cryopreservation procedures that are critical for safe, efficient archiving of the mutant collection as described by Guan et al. Nevertheless, despite the apparent richness of mouse genetic variants in the worldwide repositories, the availability of the mouse genome sequence impelled the community to tackle the challenge of developing a genome-wide comprehensive mutant resource, comprising a mouse mutant for every gene in the mouse genome. Thus, the International Knockout Mouse Consortium was born, tasked to generate mainly conditional but also constitutive alleles in over 17,000 genes in Embryonic Stem (ES) cells (Bradley et al.). The many months of bench and tissue culture work once necessary to prepare targeting vectors and select ES cells with the desired constructs are now replaced by an electronic request for individual ES cell clones that can be converted into mice within weeks. While there are still several thousand genes that need to be targeted in order to establish a complete library of mouse mutants for every proteincoding gene in the genome, the finish line is within sight. Additionally, the role of non-coding RNA, microRNAs, and regulatory elements will need to be tackled using similarly unbiased approaches. The utility of this resource is amplified by the ability to create conditional mutants, an ability that is dependent upon the quality and diversity of lines carrying appropriate Cre drivers. Murray et al. review the current state of the art of Cre driver resources along with future developments. It is important that a concomitant effort in the production of well-annotated Cre lines accompanies the global mutagenesis efforts.

One of the greatest assets for the study of mammalian biology is the detailed knowledge generated by comprehensive phenotypic characterization of a wide range of genetic traits. Classically, the repertoire of investigated phenotypes in mouse mutants was narrow and driven by specific hypotheses. However, over the last two decades, reflecting the needs of both phenotype- and gene-based large-scale screens, many centers have focused their efforts on the coordination and standardization of protocols and procedures for comprehensive phenotypic assessments. In this issue, Ayadi et al., and Fuchs et al. describe centralized programs and facilities with wide-ranging expertise in physiological, biochemical and behavioral systems. Moreover, Laughlin et al. describe a more specialized network of phenotyping centers with a focus on metabolic traits. Particularly intriguing are early insights from the initial pilots in large scale, highly standardized and comprehensive phenotyping efforts. Overall, around $80 \%$ of homozygous knockout mutant lines reveal at least one phenotype. While over $30 \%$ of these homozygous lines are not viable, the phenotypic hit rate in heterozygote alleles (of these non-viable lines) was $70 \%$. These programmes demonstrate the power of comprehensive phenotyping for defining gene function and uncovering pleiotropy. Moreover, they reinforce a growing view that the community should proceed with broad based phenotyping of mutants for every gene in the mouse genome. The International Mouse Phenotyping Consortium (IMPC) has been formed to address this challenge, and Brown \& Moore discuss the plans and progress that have been made since the consortium was formally launched in 2011 .

Undertaking large scale, genome-wide phenotyping will be an immense undertaking, but an equally difficult challenge will be the capture, annotation and dissemination of all the data generated along with its integration with other datasets, not only from mouse, but other model organisms and human. Mallon et al. describe past developments and future plans for large-scale phenotyping programmes, including the comprehensive informatics solutions required for the IMPC. These range from mouse production and tracking systems, to standardized phenotyping procedures and their data management, to data QC and annotation. Key to making progress in the area of phenotype annotation will be the development of a robust mammalian phenotype ontology and Smith and Eppig, discuss progress with the Mammalian Phenotype Ontology as a standard for mouse phenotype data. Central to the full understanding of gene function and genome interaction will be the incorporation of IMPC data into the Mouse Genome Informatics (MGI, http://www.informatics.jax.org) data resource, where the range of genetic, genomic, and biological data for mouse are placed in an integrated context. Moreover, the mapping of phenotypic traits across species will be important in order to relate phenotype discovery in mouse to biological and clinical phenotypes detected and described in other organisms. Gkoutos et al. review progress in the area of comparative phenomics, which will be critical to ensuring that we can make powerful, intelligent queries about phenotypes between multiple species.

While programmes such as the IMPC will provide an extraordinary database of baseline function for mouse genes, there remains the problem of integrating genetic variation and its contribution to phenotypic traits, thus identifying and characterizing the basis for phenotypic variation in complex traits and common diseases. A large number of well-characterized inbred strains of laboratory mice and their phenotypic diversity have been extensively utilized for the identification of physiological, metabolic, and behavioral quantitative trait loci (QTL). These studies were combined with the analysis of recombinant inbred strains and congenic lines, but the resources developed and used were often underpowered. However, there has been an 
extraordinary resurgence in the development of new approaches to dissect and analyse the genetic and phenotypic variation between inbred lines. Ghazalpour et al., describe the application of a novel association-based mixed model algorithm for the analysis of the Hybrid Mouse Diversity Panel. Chromosome substitution strains (CSSs) have had a major impact on the identification of QTLs and their underlying genetic variants, and Nadeau et al. review their role in dissecting a diverse array of genetic phenomena, such as epistasis, parent-of-origin effects and heritable epigenetic changes. Several papers in this issue provide current progress on the establishment of large multiparental panels of, first, recombinant inbred lines with fixed and reproducible genotypes-the Collaborative Cross (Welsh et al.) and, second, heterogeneous outbred mice which are unique and capture limitless combinations of segregating alleles (Churchill et al., Yalcin and Flint). The latter category includes the Diversity Outbred (DO) mice (Churchill et al.), as well as Heterogeneous Stocks (HS) and Commercial Outbreds (CO) (Yalcin and Flint). These new resources have been established with an aim to dramatically boost the level of genetic diversity and phenotypic heterogeneity, thereby increasing the resolution of mapping complex traits. The assembled reviews along with the linked publications provide early insights into the genomic architecture of these lines, and also serve as a practical guide related to their distribution and data sharing policies.

Finally, it is important to reiterate that, as we allude to above, the success and impact of these new functional genomics resources in the mouse should be considered in the wider context of mammalian biology and human disease studies. More than 2000 single-gene Mendelian diseases have been molecularly defined. However, the understanding of the genetic basis of complex common disorders lags behind. The genome-wide association studies (GWAS) of many complex diseases have uncovered a large number of common risk-associated alleles. The loci found by GWAS are mostly of small effect and explain a relatively low proportion of the heritability of complex traits. Furthermore, dense genotyping and extensive sequencing of thousands of genomes now permit near complete ascertainment of genetic variation, including low-frequency single nucleotide or copy number variants. The identification of their functional impact and validation will demand the use of model organisms. The collected resources and approaches described in this special issue (see Table 1) demonstrate the unique power of mouse genetics and genomics research to deliver a comprehensive functional map of a mammalian genome. The abundance of these new tools and resources, including phenotypically and genetically characterized mouse lines, libraries of ES cells with conditional and loss-of-function alleles, as well as candidate loci (genes and regulatory elements) identified by systems biology analysis, will be vital for the interpretation of variants identified in human and animal genetic studies. Nevertheless, there is still much to be done. Looking to the future, it is becoming clear that we need to continue to improve the richness and variety of available mouse mutant alleles and consider rapid and efficient approaches to generate and phenotype allelic combinations, all in the context of improved tools for phenotyping and annotation. This and more will be needed for a more profound mechanistic understanding of mammalian biology and disease.

Lastly, we are aware that the mammalian genome community represents the primary readership of Mammalian Genome. However, with this special issue we attempt to acquaint a broader biomedical community with the genetic toolbox of the mouse. We hope that you enjoy and benefit from this issue and will share it with your colleagues from other fields. In the future, we look forward to reading more about your research inspired by the approaches, tools and resources described here. 


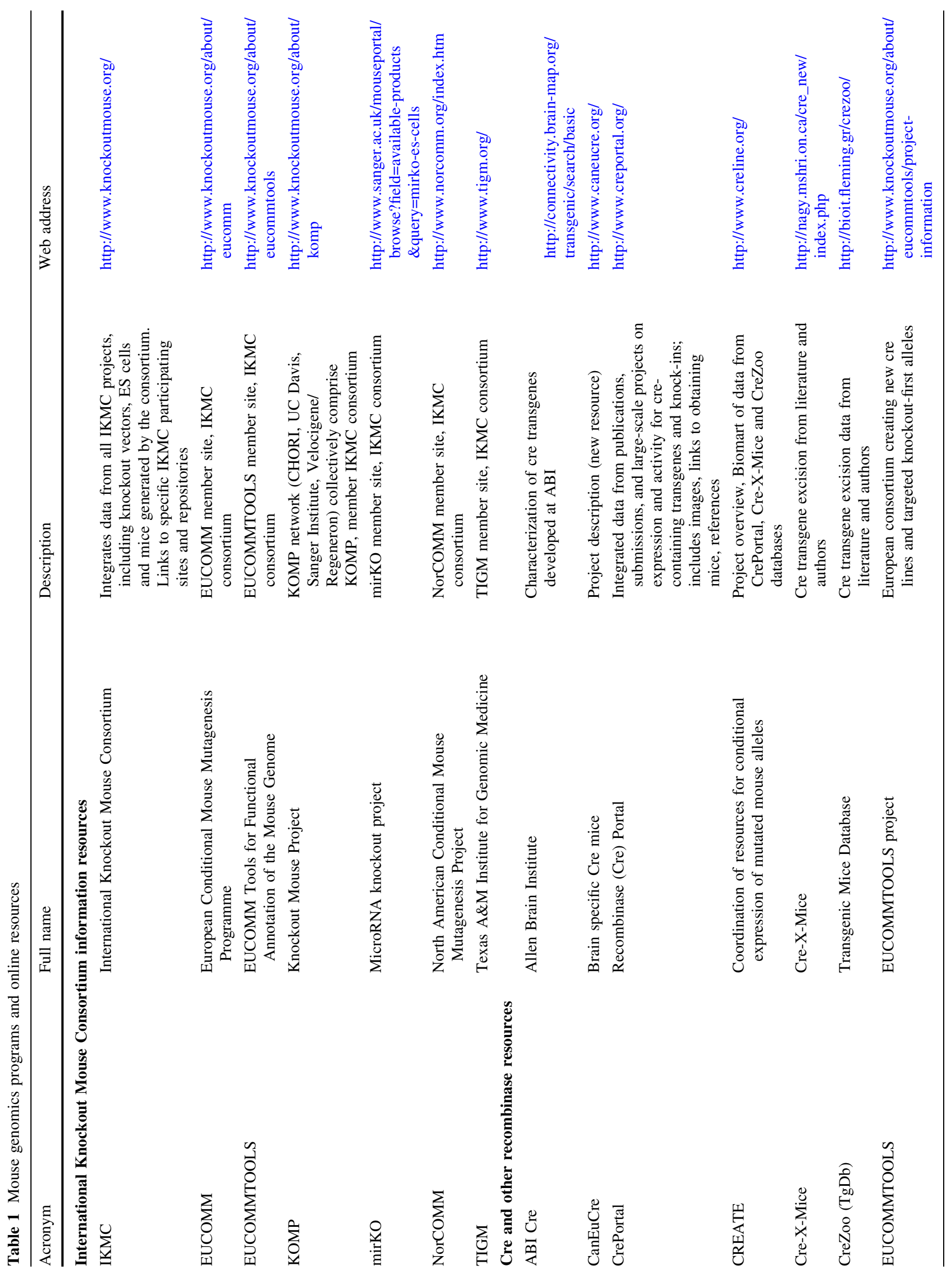




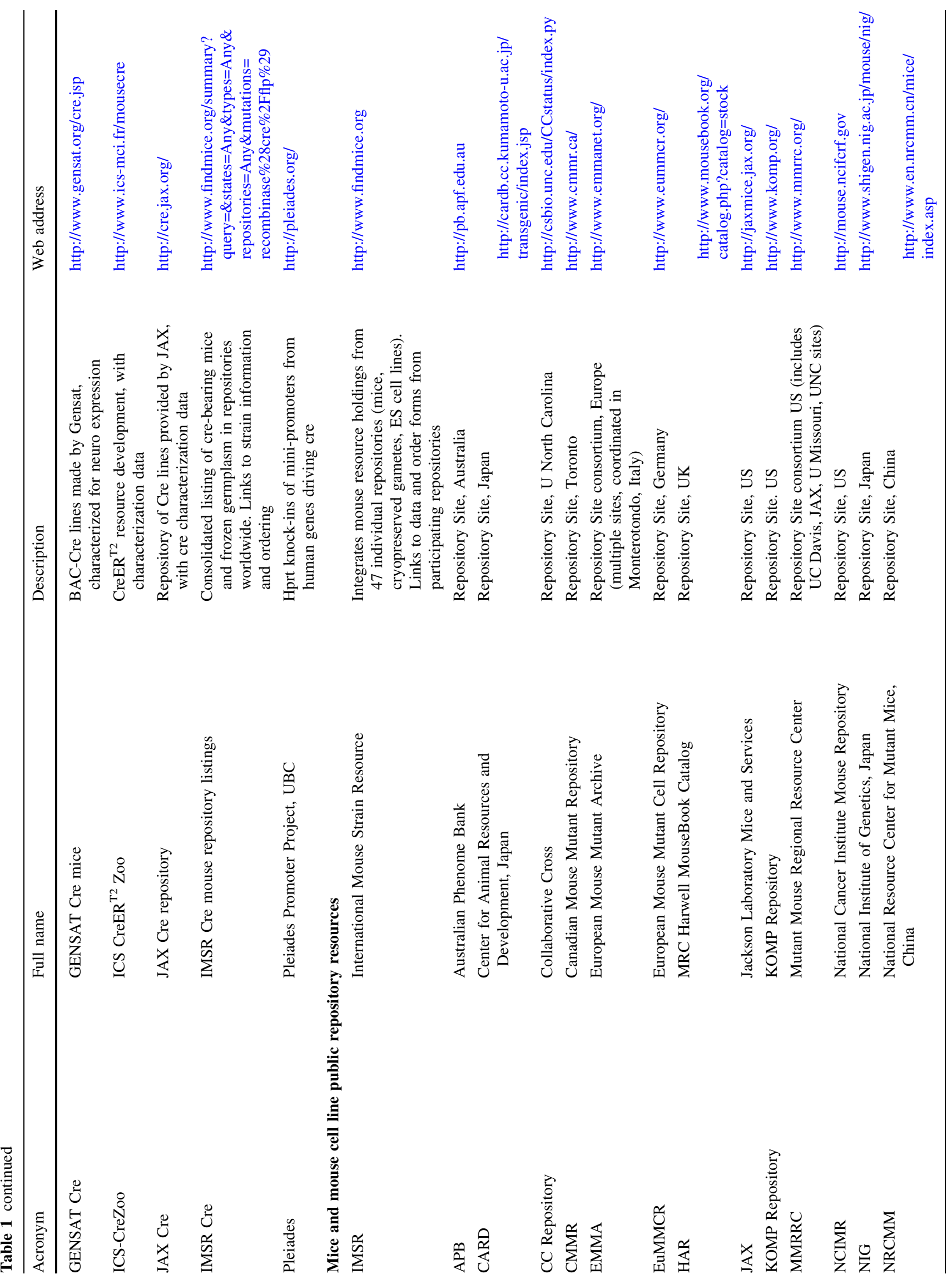




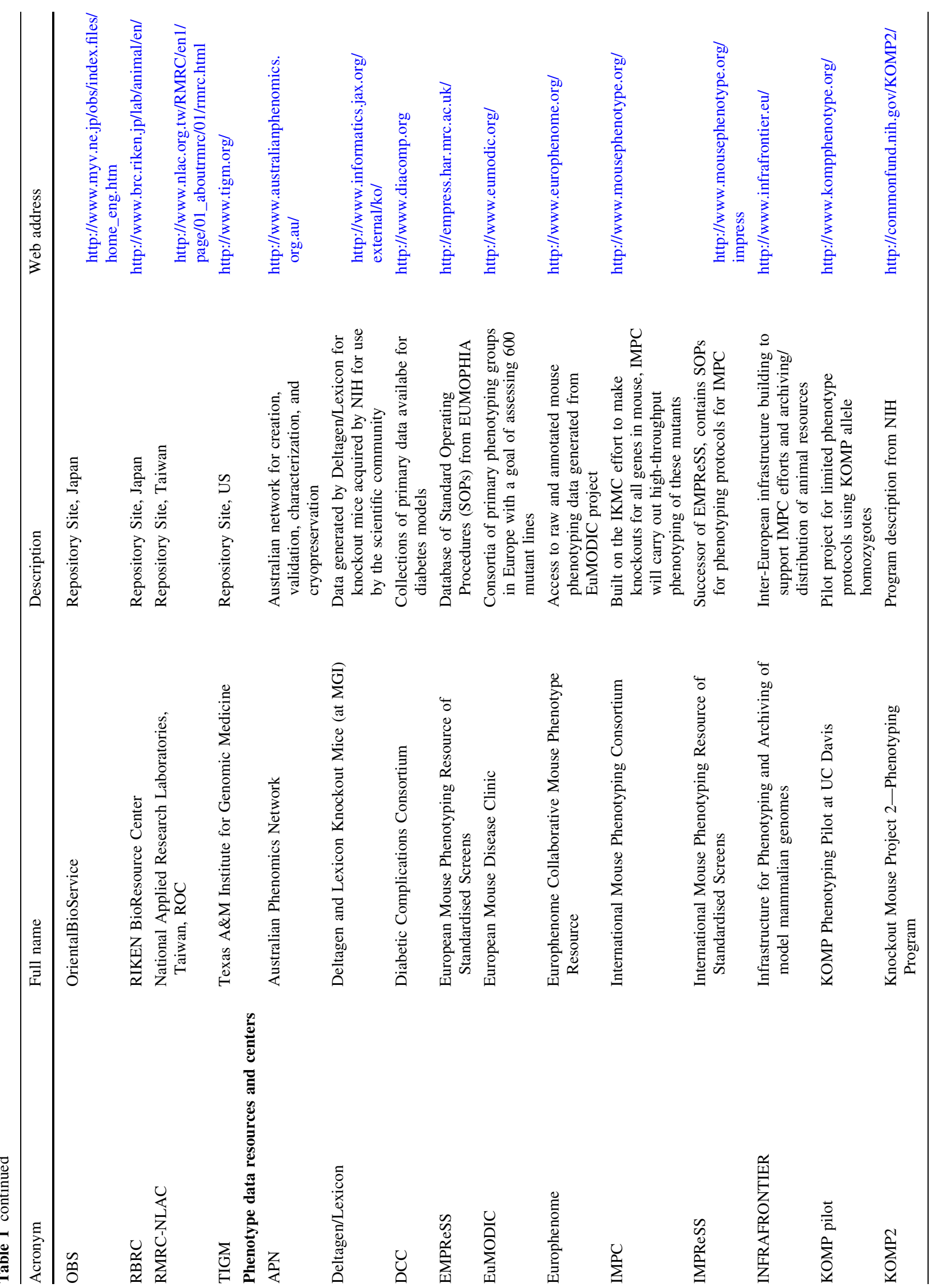




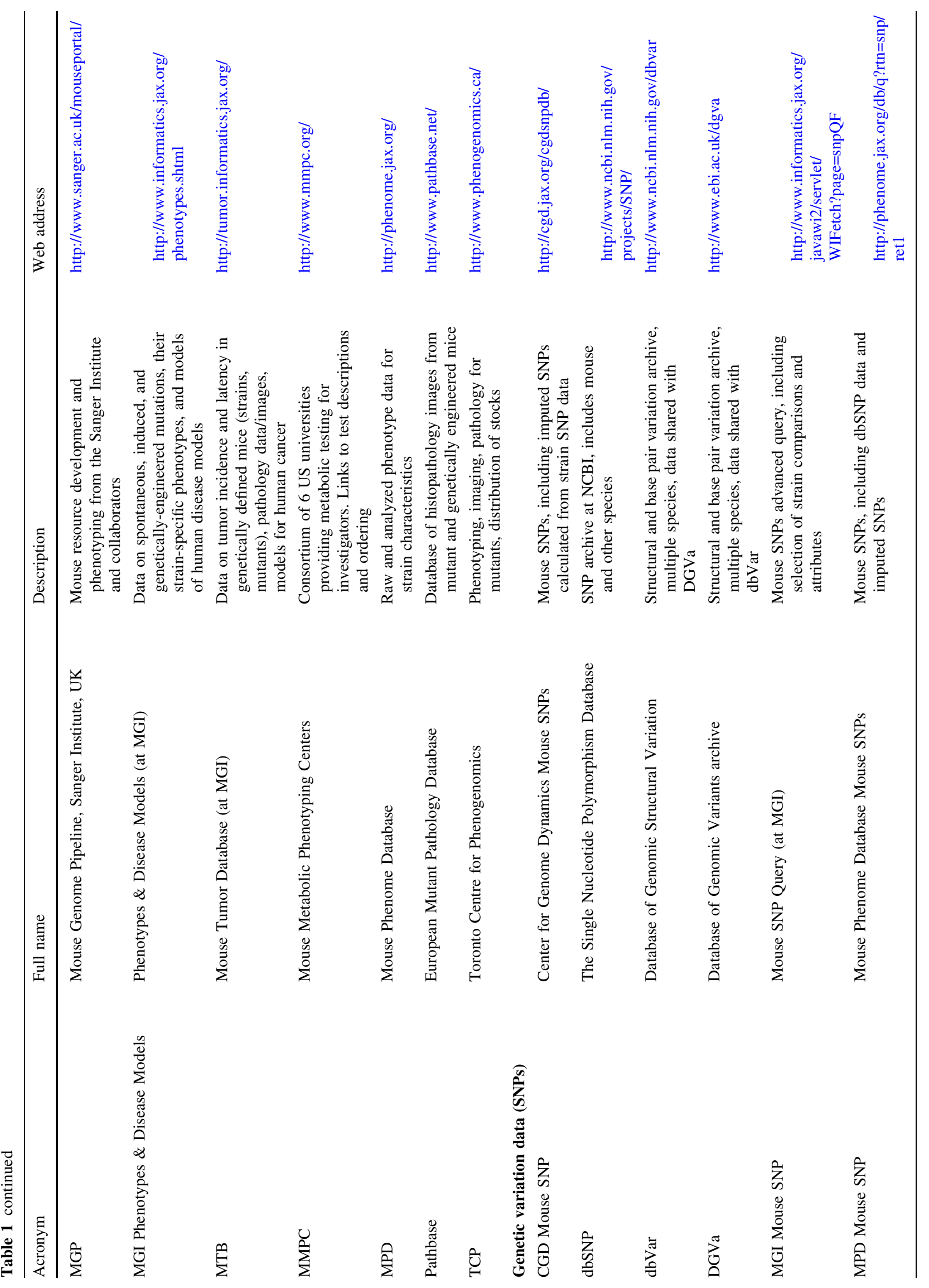




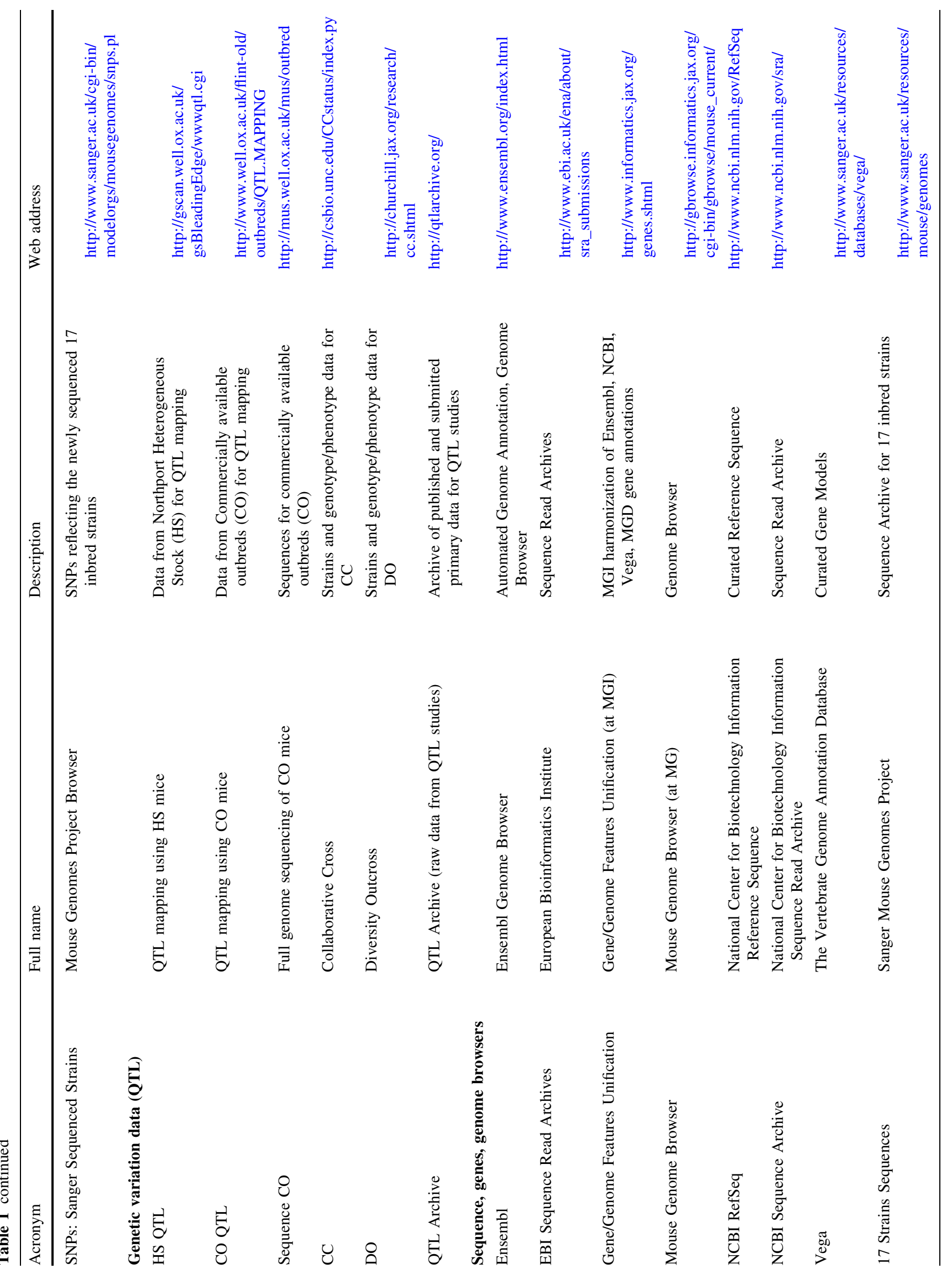




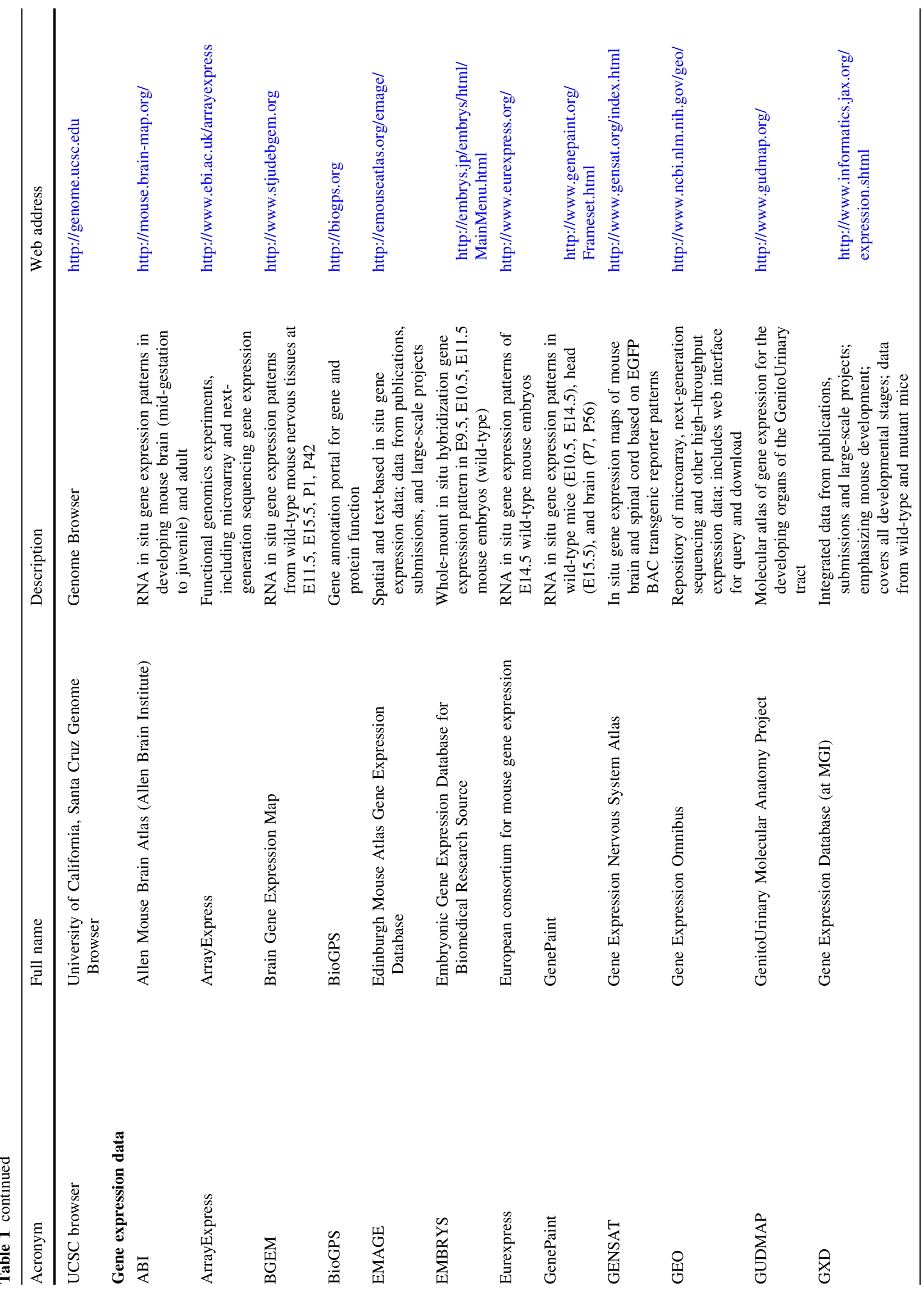




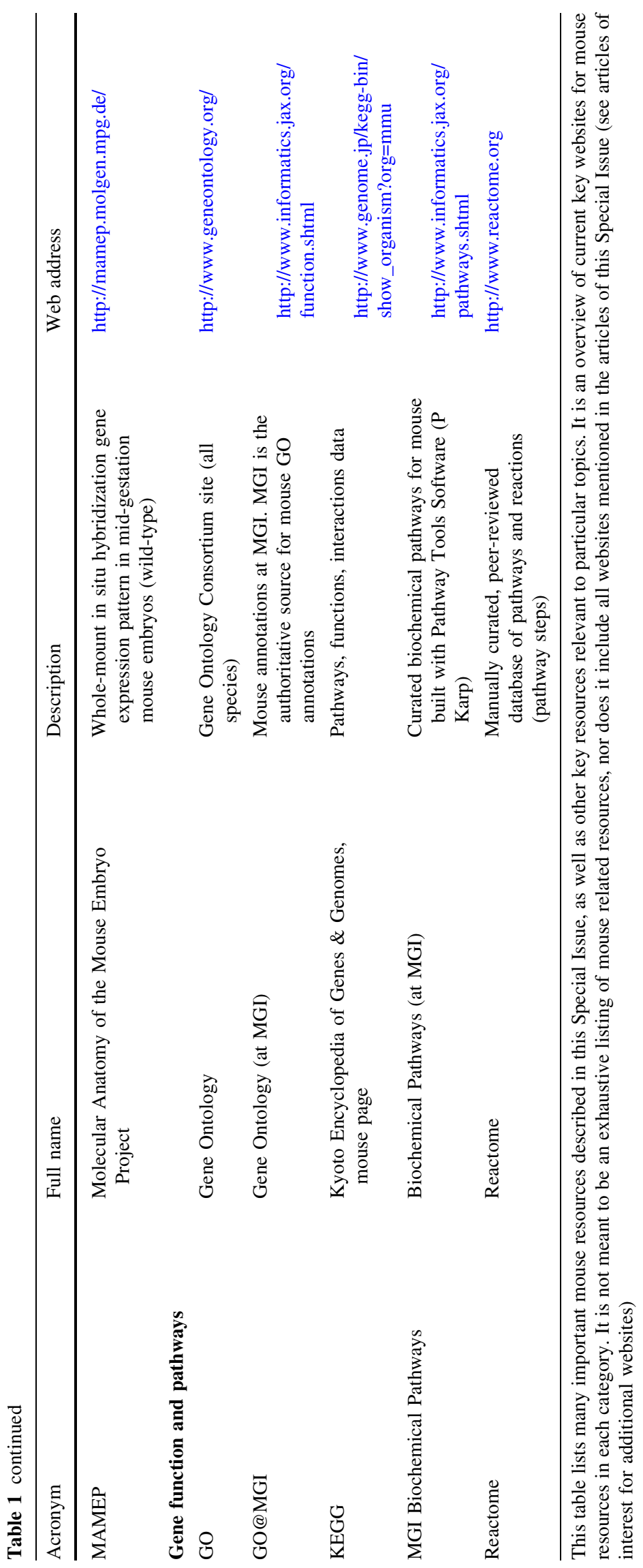




\section{References}

Keane TM, Goodstadt L, Danecek P, White MA, Wong K, Yalcin B, Heger A, Agam A, Slater G, Goodson M, Furlotte NA, Eskin E, Nellåker C, Whitley H, Cleak J, Janowitz D, Hernandez-Pliego P, Edwards A, Belgard TG, Oliver PL, McIntyre RE, Bhomra A,
Nicod J, Gan X, Yuan W, van der Weyden L, Steward CA, Bala S, Stalker J, Mott R, Durbin R, Jackson IJ, Czechanski A, Guerra-Assunção JA, Donahue LR, Reinholdt LG, Payseur BA, Ponting CP, Birney E, Flint J, Adams DJ (2011) Mouse genomic variation and its effect on phenotypes and gene regulation. Nature 477:289-294 